\title{
稀有植物四川金粟兰化学成分的进一步研究
}

\author{
王立军刘彩云* 熊 娟 胡金锋* \\ (复旦大学药学院 上海 201203)
}

\begin{abstract}
摘要 对稀有植物四川金粟兰(Chloranthus sessilifolius)全草甲醇提取物开展进一步化学成分研究，从中分离得到 2 个 二萜类、 1 个苯丙素类、 1 个黄酮类和 1 个木脂素类化合物. 利用多种波谱和光谱技术(如红外、紫外、1D/2D NMR、 ECD、HR-ESIMS)结合相关文献中的理化数据确定了它们的结构分别为: $(3 R, 5 S, 6 S, 9 S, 10 S, 13 S)-3,6,13$-三羟基-对映松香 烷-8(14)-烯-7-酮(1)、3 $\alpha, 15$-二羟基- $13 \alpha$-甲氧基-对映松香烷-8(14)-烯-7-酮(2)、 $p$-香豆酰- $\beta$-苯乙胺(3)、柚皮素三甲基醚 (4)和 $\left(3 R, 4 R, 3^{\prime} R, 4^{\prime} R\right)-6,6^{\prime}$-二甲氧基-3,4,3',4'-四氢- $2 H, 2 H^{\prime}$-[3,3']-双色原烯-4,4'-二醇(5). 其中, 化合物 1 为新的对映松香烷 型二萜, 而 $3 \sim 5$ 则均为首次从金粟兰属植物中分离得到的化合物.
\end{abstract}

关键词 四川金粟兰; 金粟兰科; 化学成分; 对映松香烷二萜

\section{Further Chemical Constituents from the Rare Chloranthaceae Plant Chloranthus sessilifolius}

\author{
Wang, Lijun Lau, Choiwan* Xiong, Juan Hu, Jinfeng* \\ (School of Pharmacy, Fudan University, Shanghai 201203)
}

\begin{abstract}
Two diterpenoids and a few miscellaneous components (one phenethylpropanoid, one flavonoid, and one lignan) were isolated from the $\mathrm{MeOH}$ extract of the whole plant of the rare Chloranthaceae plant Chloranthus sessilifolius during a further phytochemical reinvestigation. Based on their spectroscopic data (i.e., IR, UV, 1D/2D NMR, ECD, and HR-ESIMS) and/or comparing with those reported physicochemical data in related literature, the structures were determined to be $(3 R, 5 S, 6 S, 9 S, 10 S, 13 S)$-3,6,13-trihydroxy-ent-abieta-8(14)-en-7-one (1), 3 $\alpha, 15$-dihydroxy-13 $\alpha$-methoxy-ent-abieta-8(14)-en7-one (2), $p$-coumaroyl- $\beta$-phenethyl amine (3), narigenin trimethyl ether (4), and $\left(3 R, 4 R, 3^{\prime} R, 4^{\prime} R\right)$-6,6'-dimethoxy-3,4,3',4'tetrahydro- $2 H, 2 H^{\prime}-\left[3,3^{\prime}\right]$-bichromenyl-4,4'-diol (5). Among them, compound $\mathbf{1}$ is new, while compounds $3 \sim \mathbf{5}$ were isolated from the Chloranthus genus for the first time.
\end{abstract}

Keywords Chloranthus sessilifolius; Chloranthaceae; chemical constituents; ent-abietane-type diterpenoids

金粟兰科(Chloranthaceae)金粟兰属(Chloranthus) 是一个全球拥有不足 20 个种的小属, 该属植物主要分 布在亚洲的热带和温带地 ${ }^{[1]}$. 我国有 13 个种和 5 个变 种，民间多以此属植物全草(如中药及 Chloranthus serratus)入药, 具有社风散寒、舒筋强骨、活血散瘀和消肿 止痛等功效 ${ }^{[1]}$. 金粟兰属植物往往富含倍半萜单体和乌 药烷型倍半萜(lindenane)二聚体 ${ }^{[2 \sim 5]}$, 同时亦被报道代 谢产生对映贝壳烷(ent-kaurane)和半日花烷型(labdane) 二萜类化合物 ${ }^{[2]}$. 四川金粟兰 (Chloranthus sessilifolius K. F. Wu) 作为中国特有的稀有金粟兰属多年生草本植 物, 目前仅发现生长在我国四川省风栖山林下潮湿地带
${ }^{[6]}$. 我们课题组 ${ }^{[7,8]}$ 前期从四川金粟兰植物中发现一系列 具有神经抗炎活性的新型对映松香烷型(ent-abietane)二 萜类化合物. 其全草的甲醇粗提物用水混悬，再依次用 石油醚、乙酸乙酯和正丁醇进行萃取. 乙酸乙酯层 (248.0 g) 减压浓缩后, 经硅胶柱色谱得到 6 个组分, 本 工作仅将其中第三个组分(Fr.C)作为研究对象, 利用多 种色谱分离手段(如 MCI 和 HPLC)从中得到了 5 个单体 成分. 通过红外、紫外、1D/2D NMR、ECD 和 HR-ESIMS, 以及对照文献报道的理化和核磁数据, 分别鉴定为一个 新的对映松香烷型二萂 $(3 R, 5 S, 6 S, 9 S, 10 S, 13 S)-3,6,13$-三 着基-对映松香烷-8(14)-烯-7-酮(1)和 4 个已知化合物(图

*E-mail: jfhu@fudan.edu.cn

Received February 1, 2016; revised March 4, 2016; published online March 28, 2016.

Project supported by National Natural Science Foundation of China (Nos. 21472021, 81273401, 21575029) and the National Basic Research Program of China (973 Program, No. 2013CB530700).

国家自然科学基金(Nos. 21472021, 81273401, 21575029)和国家重点基础研究发展计划(973 计划, No. 2013CB530700)资助项目. 
1), 即: $13 \alpha$-甲氧基- $3 \alpha, 15$-二羟基-对映松香烷-8(14)-烯7-酮 $\left(=15\right.$-hydroxysessilifol F) (2) ${ }^{[9] 、} p$-香豆酰- $\beta$-苯乙胺 (3) ${ }^{[10]}$ 、柚皮素三甲基醚(4) ${ }^{[11]}$ 和 $\left(3 R, 4 R, 3^{\prime} R, 4^{\prime} R\right)-6,6^{\prime}$-二甲 氧基 - $3,4,3^{\prime}, 4^{\prime}$ - 四氢 - $2 H, 2 H^{\prime}-\left[3,3^{\prime}\right]$ - 双色原烯 - $4,4^{\prime}$ - 二 醇 $(5)^{[12]}$.

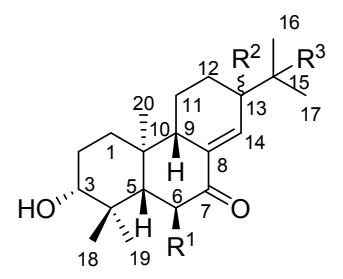

$1 \mathrm{R}^{1}=\mathrm{OH}, \mathrm{R}^{2}=\beta-\mathrm{OH}, \mathrm{R}^{3}=\mathrm{H}$

$2 \mathrm{R}^{1}=\mathrm{H}, \mathrm{R}^{2}=\alpha-\mathrm{OCH}_{3}, \mathrm{R}^{3}=\mathrm{OH}$
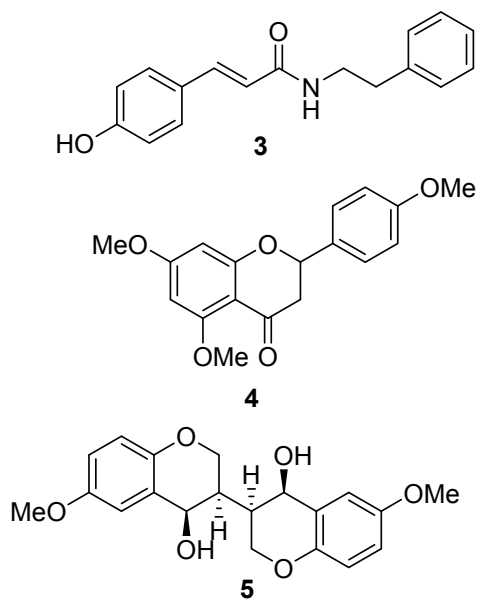

图 1 化合物 $1 \sim 5$ 的结构

Figure 1 Chemical structures of compounds $\mathbf{1} \sim \mathbf{5}$

\section{1 结果与讨论}

化合物 1 为无色无定形粉末, 其分子式 $\left(\mathrm{C}_{20} \mathrm{H}_{32} \mathrm{O}_{4}\right)$ 是根据其 HR-ESIMS(准分子离子峰检测定为 $\mathrm{m} / \mathrm{z}$ $337.2375[\mathrm{M}+\mathrm{H}]^{+}$, 计算值为 337.2373) 推断出来的, 并得到其 ${ }^{13} \mathrm{C}$ NMR 数据的佐证. 在 IR 光谱中, 3408 (br.)、1693 和 $1633 \mathrm{~cm}^{-1}$ 处吸收带显示, 该化合物依次 存在羟基和 $\alpha, \beta$ 不饱羰基官能团, 后者得到 UV 光谱 $\left(\lambda_{\max }\right)$ 在 $248.5 \mathrm{~nm}$ 吸收带的进一步确认. 分析其 ${ }^{1} \mathrm{H}$ NMR 数据, 推断化合物 1 结构中含有 3 个单峰甲基 $(\delta$ $0.98,1.10,1.31$ ), 2 个双峰甲基 $[\delta 0.95,0.99$ (each d, $J=$ $7.0 \mathrm{~Hz}, \mathrm{Me}-16, \mathrm{Me}-17)] 、 1$ 个烯氢 $[\delta 6.72$ (br s, H-14)]和 2 个连氧的次甲基 $[\delta 3.30(\mathrm{dd}, J=11.7,4.2 \mathrm{~Hz}, \mathrm{H}-3), 4.28$ (d, $J=12.6 \mathrm{~Hz}, \mathrm{H}-6)$ ]等质子信号. 其 ${ }^{13} \mathrm{C} \mathrm{NMR}$ 谱图中的 20 个碳信号可归属为 1 个羰基, 5 个甲基, 4 个亚甲基, 6 个次甲基(包括 2 个分别在 $\delta 78.8$ 和 75.2 的含氧次甲基; 1 个在 $\delta 141.9$ 的双键次甲基), 1 个连氧的叔碳 ( $\delta 72.4)$ 和 3 个季碳(包括 1 个双键季碳在 $\delta$ 135.9). 以上数据说明 化合物 1 在结构上与从宽叶金粟兰 $(C$. henryi $)$ 中分离得
到的对映松香烷二萜类化合物 15-hydroxysessilifol $\mathrm{F}^{[9]}$ (化合物 2)十分相似, 区别在于化合物 1 少了 1 个甲 氧基信号和 1 个连氧的叔碳信号, 取而代之的是 $\mathbf{1}$ 中多 了 1 个连氧的次甲基信号, 表明化合物 1 中 3 个羟基都 是游离状态, 且位置发生了变化. 在化合物 1 的 COSY 谱中，除看到自旋体系 H-5-H-6 外，还可看到异丙基的 两个甲基 Me-16(17)与 H-15 相关. 以上数据很容易证实 化合物 1 中的 C-6 位(而非 C-15) 发生氧化而连有一个 差弪基. 在 1 的 HMBC 谱图上, H-6 [ $\delta 4.28,(\mathrm{~d}, J=12.5 \mathrm{~Hz}$, H-6 $\alpha$ )]同 C-5 ( $\delta$ 55.6)/C-7 ( $\delta$ 202.5)相关可以进一步证实 C-6 被着化(图 2). 化合物 1 的相对构型是通过分析相关 质子的偶合常数以及 NOESY 谱确定的. H-3 和 H-6 显示 大的偶合常数 $\left(J_{\mathrm{H}-2 \alpha, 3}=11.1 \mathrm{~Hz} ; J_{\mathrm{H}-5,6}=12.5 \mathrm{~Hz}\right)$ 证实这 两个质子都处在直立键。在 $\operatorname{NOESY}($ 图 2) 谱中, Me-18/H-3, Me-18/H-5, Me-19/H-6, H-3/H-1 $\beta$, H-1 $\beta / \mathrm{H}-9$, $\mathrm{H}-9 / \mathrm{H}-12 \beta$ 和 H-12 $\alpha / \mathrm{Me}-16(17)$ 等 NOE 相关信号确定了 H-3、H-5、H-9、OH-6 和 OH-13 都是处于 $\beta$-位. 化合物 1 的绝对构型是通过与我们前文报道的一个结构类似的 对映松香烷型二萜 sessilifol $\mathrm{D}^{[7]}$ 比对它们的圆二色谱 (ECD)加以确定的. 化合物 1 在 259 和 $333 \mathrm{~nm}$ 两处均产 生正的 Cotton 效应与 sessilifol D (254 和 $330 \mathrm{~nm})^{[7]}$ 一致. 因此，化合物 1 被确定为 $(3 R, 5 S, 6 S, 9 S, 10 S, 13 S)-3,6,13-$ 三 羟基 - 对映松香烷 -8(14)- 烯 -7-酮 (1) [3R, $5 S, 6 S, 9 S, 10 S$, 13S)-3,6,13-trihydroxy-ent-abieta-8(14)-en-7-one](图 1).
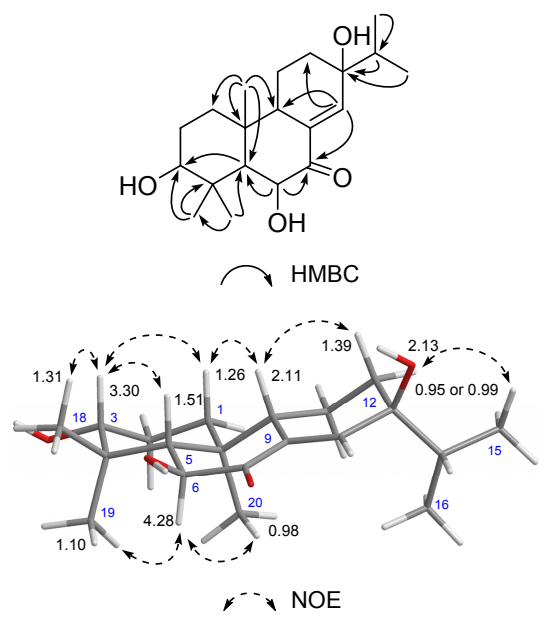

图 2 化合物 $\mathbf{1}$ 的 $\mathrm{HMBC}$ 和 $\mathrm{NOE}$ 相关.

Figure 2 Key HMBC and NOE correlations of 1

在前期工作中, 我们 ${ }^{[7,8]}$ 发现部分对映松香烷型二 萜，如 sessilifol F、sessilifol I、 $3 \alpha, 7 \beta$-dihydroxy-ent-abieta$8,11,13$-triene, 具有很好的神经抗炎活性 $\left(\mathrm{IC}_{50}\right.$ 分别为 8.3、7.4、4.3 $\mu \mathrm{mol} / \mathrm{L})$. 本工作中, 在神经抗炎模型 BV-2 上对所分离得到的两个对映松香烷型二萜 $(1,2)$ 进行了 类似的活性测试, 但两者活性都很弱 $\left(\mathrm{IC}_{50}>50 \mu \mathrm{mol} / \mathrm{L}\right)$. 


\section{2 实验部分}

\section{1 仪器与试剂}

比旋光值采用 Rudolph Autopol VI 旋光仪测定; 紫 外和红外分别采用日立 U-2900E 双光束分光光度仪和 Thermo Scientific Nicolet iS5 傅里叶变换红外光谱仪测 定; 圆二色谱采用 JASCO-810 分光偏振计测定; 核磁共 振谱采用 Bruker Avance III $400 \mathrm{MHz}$ 和 $600 \mathrm{MHz}$ 核磁共 振仪测定, 化学位移以 $\delta$ 表示并以相应的残余溶剂峰作 为参照; 低分辨质谱采用 Agilent 1100 series 质谱仪测 定; 高分辨质谱则采用 Agilent Technologies 6224 TOF LC/MS 质谱仪测定; 半制备型高效液相色谱仪为 Waters e2695 系列, 配备有 Waters 2998 PDA 检测器和 Waters 2424 ELSD 检测器; 半制备型色谱柱为 SunFire ODS column ( $5 \mu \mathrm{m}, 250 \mathrm{~mm} \times 10 \mathrm{~mm}$; 流速: $3.0 \mathrm{~mL} / \mathrm{min})$; 正 相柱色谱硅胶规格为 200 300 目(烟台牟平区康必诺化 学试剂厂和青岛海洋化工厂分厂); 三菱凝胶吸附树脂 MCI gel CHP20P 规格为 75 150 $\mu \mathrm{m}$ (Mitsubishi Chemical Industries, 日本东京); 薄层层析 (TLC) 板规格为 GF254/0.25 mm(烟台牟平区康必诺化学试剂厂). 分析 纯试剂购自于上海泰坦科技股份有限公司，色谱级溶剂 购自北京百灵威科技有限公司, 氛代试剂由 Sigma-Aldrich 生产; 薄层层析显色剂为 $10 \%$ $\mathrm{H}_{2} \mathrm{SO}_{4} / \mathrm{EtOH}(V: V)\left(120{ }^{\circ} \mathrm{C}\right.$ 烘烤).

四川金粟兰(Chloranthus sessilifolius) 全草 2012 年 8 月采集于四川风栖山, 经第二军医大学生药教研室黄 宝康教授鉴定. 标本(20120801)现存于复旦大学药学院 天然药物化学教研室.

\section{2 提取与分离}

四川金粟兰全草 $6.8 \mathrm{~kg}$, 用 $20 \mathrm{~L}$ 的纯甲醇室温冷浸 $24 \mathrm{~h}$, 共 3 次. 合并 3 次提取液, 经过滤、减压浓缩, 得 到 $463 \mathrm{~g}$ 总浸亳. 将得到的浸亳用 $2 \mathrm{~L}$ 的水分散制成悬 浊液, 然后依次用石油醚、乙酸乙酯和正丁醇进行萃取. 乙酸乙酯部分经减压浓缩得 $248 \mathrm{~g}$ 样品, 接着用硅胶柱 色谱 $(10 \mathrm{~cm} \times 80 \mathrm{~cm})$ 进行初步分离, 石油醚和丙酮梯度 洗脱 $(9: 1 \sim 0: 1, V / V)$, 得到 6 个组分 Fr.A $\sim$ Fr.F 前文 已报道从 Fr.A、Fr.B、Fr.D、Fr.E 等组分中分离得到一 系列二萜 ${ }^{[7,8]}$ 和倍半萜 ${ }^{[8]}$. Fr.C 和 Fr.F 初步检测分析时原 以为不含感兴趣的二萜成分, 被置于一旁未做研究 ${ }^{[8]}$. 后经仔细分析发现 Fr.C 含有少量二萜成分, 但 Fr.F 是从 硅胶柱色谱上最后洗脱下来的组分, 仅十几毫克且色素 较重, 经 TLC 和 HPLC 分析发现不含任何感兴趣的化学 成分, 被最终弃置.

Fr.C 采用 $\mathrm{MCI}$ 柱色谱 $(6.0 \mathrm{~cm} \times 30 \mathrm{~cm})$ 进行进一步 分离，洗脱剂为甲醇和水 $(50 \% \sim 100 \%, V / V)$. 将 $70 \% \sim$
$80 \%$ 甲醇部分 $(F r . C B)$ 用硅胶柱 $(4 \mathrm{~cm} \times 60 \mathrm{~cm})$ 进行梯度 洗脱, 洗脱剂为石油醚和乙酸乙酯 $(4: 1 \sim 1: 1, V / V)$ 得 到 5 个组分 Fr.CB1 Fr.CB5. 将 Fr.CB2 用硅胶柱色谱 $(2.5 \mathrm{~cm} \times 40 \mathrm{~cm})$ 分离纯化, 洗脱剂为二氯甲烷和乙酸 乙酯 (1：1, V/V), 再用半制备 HPLC[ 流动相: $\left.V(\mathrm{MeOH}): V\left(\mathrm{H}_{2} \mathrm{O}\right)=65: 35\right]$ 进一步纯化得到化合物 3 $\left(5.5 \mathrm{mg}, t_{\mathrm{R}}=10.1 \mathrm{~min}\right)$ 和化合物 $5\left(4.1 \mathrm{mg}, t_{\mathrm{R}}=6.8 \mathrm{~min}\right)$. 化合物 $4\left(7.8 \mathrm{mg}, t_{\mathrm{R}}=15.5 \mathrm{~min}\right)$ 是经过一系列的硅胶柱 色谱最后经半制备 HPLC 色谱 [流动相: $V(\mathrm{MeOH})$ : $\left.V\left(\mathrm{H}_{2} \mathrm{O}\right)=55: 45\right]$ 从 Fr.CB3 获得. Fr.CB5 用半制备 HPLC 色谱[流动相: $V(\mathrm{MeOH}): V\left(\mathrm{H}_{2} \mathrm{O}\right)=55: 45$ ] 进行纯化, 最后得到化合物 $\mathbf{1}\left(2.1 \mathrm{mg}, t_{\mathrm{R}}=12.1 \mathrm{~min}\right)$ 和化合物 $\mathbf{2}(2.6$ $\left.\mathrm{mg}, t_{\mathrm{R}}=19.2 \mathrm{~min}\right)$.

(3R,5S,6S,9S, 10S, 13S)-3,6,13-三羟基-对映松香烷8(14)- 烯-7-酮 (1): 无定形粉末; $[\alpha]_{\mathrm{D}}^{25}+9.0$ (c 0.21 , $\left.\mathrm{CH}_{2} \mathrm{Cl}_{2}\right)$; UV-Vis $\left.(\mathrm{MeOH}) \lambda_{\max }\left[\log \varepsilon /\left(\mathrm{L} \cdot \mathrm{mol}^{-1} \cdot \mathrm{cm}^{-1}\right)\right]\right)$ : 248.5 (3.2); ECD $\left(c 1.19 \times 10^{-3} \mathrm{~mol} / \mathrm{L}, \mathrm{MeOH}\right)(\Delta \varepsilon): 259$ $(+1.7), 333(+0.4) \mathrm{nm} ;{ }^{1} \mathrm{H}$ NMR $\left(\mathrm{CDCl}_{3}, 400 \mathrm{MHz}\right) \delta$ : 1.74 (ddd, $J=13.4,3.5,3.5 \mathrm{~Hz}, \mathrm{H}-1 \alpha$ ), 1.26 (ddd, $J=13.4$, $13.4,3.4 \mathrm{~Hz}, \mathrm{H}-1 \beta), 1.66 \sim 1.76$ (m, H-2 $\alpha$ ), $1.56 \sim 1.67$ (m, H-2 $), 3.30$ (dd, $J=11.1,4.0 \mathrm{~Hz}, \mathrm{H}-3), 1.51$ (d, $J=12.5$ Hz, H-5 $), 4.28$ (d, $J=12.5$ Hz, H- $6 \alpha$ ), 2.06 2.16 (m, $\mathrm{H}-9 \beta), 1.70 \sim 1.80(\mathrm{~m}, \mathrm{H}-11 \alpha), 1.36 \sim 1.46(\mathrm{~m}, \mathrm{H}-11 \beta)$, $2.08 \sim 2.18(\mathrm{~m}, \mathrm{H}-12 \alpha), 1.34 \sim 1.44$ (m, H-12ß), 6.72 (br s, H-14), 0.95 (d, $J=7.0 \mathrm{~Hz}, \mathrm{Me}-16), 0.99$ (d, $J=7.0 \mathrm{~Hz}$, Me-17), 1.31 (s, Me-18), 1.10 (s, Me-19), 0.98 (s, Me-20); ${ }^{13} \mathrm{C}$ NMR $\left(\mathrm{CDCl}_{3}, 100 \mathrm{MHz}\right) \delta: 37.0(\mathrm{C}-1), 26.7(\mathrm{C}-2)$, 78.8 (C-3), 39.7 (C-4), 55.6 (C-5), 75.2 (C-6), 202.5 (C-7), 135.9 (C-8), 50.1 (C-9), 35.8 (C-10), 20.7 (C-11), 32.8 (C-12), 72.4 (C-13), 141.9 (C-14), 37.4 (C-15), 16.7 (C-16), 16.8 (C-17), 30.3 (C-18), 15.1 (C-19), 16.0 (C-20); IR (film) v: 3408 (br), 2965, 2931, 2871, 1693, 1633, 1446, 1380, 1249, $1001 \mathrm{~cm}^{-1}$; ESIMS m/z: $337.0[\mathrm{M}+\mathrm{H}]^{+}$, $359.0[\mathrm{M}+\mathrm{Na}]^{+}$; HR-ESIMS calcd for $\mathrm{C}_{20} \mathrm{H}_{33} \mathrm{O}_{4}[\mathrm{M}+\mathrm{H}]^{+}$ 337.2373 , found 337.2375 .

辅助材料(Supporting Information) 新化合物 1 的 $1 \mathrm{D} / 2 \mathrm{D} N \mathrm{NMR}$ 、高分辨质谱及 $\mathrm{ECD}$ 光谱等谱图. 这些材 料可以免费从本刊网站(http://sioc-journal.cn/)上下载.

\section{References}

[1] Editorial committee Flora of China, Science Press, Beijing, 1982, Vol. 20, pp. 80 96 (in Chinese).

(编委会, 中国植物志, 科学出版社, 北京, 1982, Vol. 20, pp. $80 \sim 96$.)

[2] Wang, A. R.; Song, H. C.; An, H. M.; Huang, Q.; Luo, X.; Dong, J. Y. Chem. Biodiversity 2015, 2, 451 . 
[3] Wang, L. J.; Xiong, J.; Liu, S. T.; Liu, X. H.; Hu, J.-F. Chem. Biodiversity 2014, 11, 919.

[4] Liu, S. T.; Xiong, J.; Tang, Y.; Wang, W. X.; Bui, V. B.; Ma, G. L.; Huang, Y.; Zhao, Y.; Yang, G. X.; Hu, J.-F. Chem. Biodiversity 2014, 11, 904.

[5] Xiong, J.; Liu, S. T.; Tang, Y.; Wang, W. X.; Bui, V. B.; Zhao, Y.; Fan, H.; Yang, G. X.; Hu, J.-F. Phytochem. Lett. 2013, 6, 586.

[6] Wang, D. Q. Lishizhen Med. Mater. Med. Res. 1991, 2, 140 (in Chinese).

(王德群, 时珍国药研究, 1991, 2, 140.)

[7] Wang, L. J.; Xiong, J.; Liu, S. T.; Pan, L. L.; Yang, G. X.; Hu, J.-F.
J. Nat. Prod. 2015, 78, 1635.

[8] Wang, L. J.; Xiong, J.; Lau, C.; Pan, L. L.; Hu, J.-F. J. Asian Nat. Prod. Res. 2015, 17, 1220.

[9] Xie, C. F.; Sun, L. M.; Liao, K. Liu, S.; Wang, M. C.; Xu, J.; Bartlam, M.; Guo, Y. Q. J. Nat. Prod. 2015, 78, 2800.

[10] Sinz, A.; Matusch, R.; Van Eläcker, F.; Santisuk, T.; Chaichana, S.; Reutrakul, V. Phytochemistry 1999, 50, 1069.

[11] Seidel, V.; Bailleul, F.; Waterman, P. G. Phytochemistry 2000, 55, 439.

[12] Sielinou, V. T.; Vardamides, J. C.; Nkengfack, A. E.; Laatsch, H. Phytochem. Lett. 2012, 5, 409.

(Qin, X.) 